\title{
Equation for the potential of an electron system with slowly varying density in the energy-functional formalism
}

\author{
Emilio Santos \\ Departamento de Física Teórica, Universidad de Santander, Santander, Spain \\ Matilde Leal \\ Escuela Universitaria del Profesorado, Universidad de Santander, Santander, Spain
}

(Received 16 April 1982)

\begin{abstract}
A second-order differential equation is derived for the electric potential of an electron system with slowly varying density. It includes consistently the first-gradient corrections to both the kinetic energy and the exchange energy. In the regime of high density, the equation reduces to the one derived by Schwinger [Phys. Rev. A 24, 2353 (1981)].
\end{abstract}

The starting point of the energy-density - functional formalism is the theorem of Hohenberg and Kohn. ${ }^{1}$ It states that there exists a functional of the electron density which is a minimum for the ground state of the system and this minimum value corresponds to the energy of that state.
The functional is not known in general, but there are several regimes for which an approximate form can be derived. One of these is the system with a slowly varying density. In this case, the functional can be generated by gradient expansion techniques and it can be written, in atomic units $(\hbar=e=m=1)$,

$$
E[\rho]=\frac{1}{2} \int \rho(\overrightarrow{\mathrm{r}}) \rho\left(\overrightarrow{\mathrm{r}}^{\prime}\right)\left|\overrightarrow{\mathrm{r}}-\overrightarrow{\mathrm{r}}^{\prime}\right|^{-1} d^{3} r d^{3} r^{\prime}+\int\left[W(\overrightarrow{\mathrm{r}}) \rho(\overrightarrow{\mathrm{r}})+F(\rho)+\epsilon G(\rho)(\vec{\nabla} \rho)^{2}\right] d^{3} r+o(\epsilon),
$$

where $\rho$ is the number of the electrons per unit volume (i.e., $\rho \geqslant 0$ ) and $W(\vec{r})$ is the external Coulomb potential energy of an electron at $\vec{r}$. The parameter $\epsilon$ is introduced as a formal expansion parameter (at the end we shall put $\epsilon=1)$ and $o(\epsilon)$ means terms of order higher than $\epsilon$. The function $F(\rho)$ has been evaluated from the homogeneous electron gas theory to be

$F(\rho)=(3 / 10)\left(3 \pi^{2}\right)^{2 / 3} \rho^{5 / 3}-(3 / 4)(3 / \pi)^{1 / 3} \rho^{4 / 3}+F_{c}(\rho)$.

The first term gives the well-known Thomas-Fermi expression, the second one the exchange (Dirac) term, and $F_{c}$ is the correlation energy. The correlation energy has been derived in the limit of high density ${ }^{2}$ and several approximate expressions are available, which are valid also for lower densities. $^{3}$ The function $G(\rho)$ is

$G(\rho)=(1 / 72) \rho^{-1}-(7 / 432 \pi)\left(3 \pi^{2}\right)^{-1 / 3} \rho^{-4 / 3}+G_{c}(\rho)$.

The first term has been derived by Kompaneets and Pavloskii, ${ }^{4}$ Kirzhnits, ${ }^{5}$ and others. The second term has been obtained recently with similar techniques. ${ }^{6,7}$ The third term (gradient correction to the correlation energy) is also known. ${ }^{7}$ This form of the functional has been used (with empirical corrections of the coefficients of the gradient terms) for the discussion of atomic and polyatomic (as well as nuclear) systems.

Functional (1), with $\epsilon=0$, gives rise to the local density approximation (LDA). The condition that $E[\rho]$ is a minimum, with a fixed number of electrons, leads to a variational problem whose Euler differential equation is a second-order one. It allows the calculation of the electron density and generalizes the early Thomas-Fermi equation. ${ }^{8}$ However, if one goes beyond LDA by retaining the terms of order $\epsilon$ in the functional (1), the Euler equation of the variational problem is an integro-differential one. It can be transformed into a fourth-order differential equation by introducing the total potential $V$ through the Poisson equation, valid outside the atomic nuclei,

$$
\nabla^{2} V=-4 \pi \rho+\nabla^{2} W=-4 \pi \rho
$$

(note that $\rho$ is the number of electrons per unit volume while $V$ and $W$ are potential energies, i.e., electrostatic potentials times the electron charge, which is -1 in atomic units). The purpose of this paper is to show that this equation can be transformed into another one with the same degree of approximation (i.e., first order in $\epsilon$ ), but with second derivatives only. The point is that it is not profitable to use a fourth-order equation (widely used in fact) if there is a second-order one which has the same degree of accuracy.

At this moment it is appropriate to call attention to the convenience of changing from the use of functionals of the density to functionals of the potential. It is a trivial extension of the Hohenberg-Kohn theorem that there is a universal functional of the potential whose minimum corresponds to the ground-state energy. This follows from the fact that the potential is uniquely related to the density through Poisson Eq. (4). A functional of the potential may be simpler than the corresponding one of the density, owing to the property that the density at a point is related locally to the potential, while the converse is not true. Therefore, potential functionals are likely more local than density functionals. A functional of the potential for atoms which has been proposed recently by Schwinger ${ }^{9}$ has allowed him to calculate the quantum correction of relative order $Z^{-2 / 3}$ to the total energy of the Thomas-Fermi atom. We shall obtain Schwinger's equation as a particular case (the high-density limit) of our equation for the potential. Besides, we show that it has the same degree of approximation as the Thomas-Fermi-Dirac-Weizsäcker theory, a fact already noted by Schwinger. ${ }^{9}$

After that, the main result of this paper can be expressed 
as a theorem. The Euler equation of the variational problem, stating that $E[\rho]$ given by Eq. (1) is stationary, agrees to order $\epsilon$ with the differential equation

$$
\nabla^{2} \bar{V}+S(\bar{V})=0
$$

in the region without external charges. The functions $\bar{V}(\overrightarrow{\mathrm{r}})$ and $S(\bar{V})$ are defined as follows. In the first place we define $V$ such that its zero coincides with the Fermi energy $E_{F}$. Then, we define the function $f(V)$ by

$$
\frac{d F(\rho)}{d \rho}=-V \leftrightarrow \rho=f(V),
$$

where $F(\rho)$ is given by Eq. (2). [The possibility of writing $\rho$ as a function of $V$ rests upon the convexity of the function $F(\rho)]$. Now, the function $\bar{V}(\vec{r})$ is defined by

$$
\bar{V} \equiv V-4 \pi \epsilon \int_{0}^{V} G(f(\gamma))\left(\frac{d f(\gamma)}{d \gamma}\right)^{2} d \gamma
$$

with $G(\rho)$ given by Eq. (3). Finally, the function $S(\bar{V})$ is given by

$$
S(\bar{V}) \equiv 4 \pi f(V)\left[1+4 \pi \epsilon G(f(V))\left(\frac{d f(V)}{d V}\right)^{2}\right]
$$

together with Eq. (7), where only terms of order $\epsilon$ should be retained.

The proof of the theorem follows. The variational problem constrained by the condition which fixes the number of electrons gives the Euler equation

$$
-V=\frac{d F(\rho)}{d \rho}-\epsilon \frac{d G}{d \rho}(\nabla \rho)^{2}-2 \epsilon G \nabla{ }^{2} \rho+o(\epsilon) .
$$

We must show that this equation agrees with Eq. (5) to first order in $\epsilon$. Equation (9) agrees with Eq. (6) to zeroth order, so that $f(V)$ can be substituted instead of $\rho$ in all first-order terms. In the resulting equation, we may obtain $\rho$ as a functional of $V$, which gives the following expression, again equivalent to Eq. (9) to first order in $\epsilon$ :

$$
\rho=f-\epsilon \frac{d f}{d V}\left(\frac{d G}{d f}(\nabla f)^{2}+2 G \nabla^{2} f\right)+o(\epsilon) .
$$

Here $G$ is a function of $f, f$ a function of $V$, and $V$ a function of $\vec{r}$. Now, taking Eq. (4) into account, it is straightforward to obtain

$$
\nabla^{2} \bar{V}=-4 \pi f+4 \pi \epsilon G\left(\frac{d f}{d V}\right)^{2} \nabla^{2} V+o(\epsilon),
$$

where $\bar{V}$ is the function defined by Eq. (7). Finally, taking Eqs. (4) and (6) into account, we substitute

$$
-\nabla^{2} V=4 \pi f(V)
$$

in the last term of Eq. (11) and we obtain Eq. (5) in the regions where the external charges vanish. This completes the proof.

The function $S(\bar{V})$ of Eq. (6) is rather involved (although straightforward) and it will not be written here explicitly. However, there is a regime where $S(\bar{V})$ is rather simple, namely, the high-density limit. For high densities, Eqs. (2) and (3) can be approximated by

$$
\begin{aligned}
& F(\rho)=(3 / 10)\left(3 \pi^{2}\right)^{2 / 3} \rho^{5 / 3}-\mu(3 / 4)(3 / \pi)^{1 / 3} \rho^{4 / 3}, \\
& G(\rho)=(1 / 72) \rho^{-1},
\end{aligned}
$$

where we have introduced $\mu$ in the second term of $F(\rho)$ as a new formal expansion parameter (which will be put equal to 1 at the end). To first order in both $\epsilon$ and $\mu$ we obtain

$$
\begin{aligned}
& \bar{V}=V+\epsilon(\sqrt{2} / 6 \pi)(-V)^{1 / 2}, \\
& \nabla^{2} \bar{V}=-(8 \sqrt{2} / 3 \pi)(-\bar{V})^{3 / 2}+\left(8 / 9 \pi^{2}\right)(9 \mu+2 \epsilon) \bar{V} .
\end{aligned}
$$

These equations, with $\epsilon=\mu=1$, have been derived by Schwinger, ${ }^{9}$ which suggested that they might extrapolate the Thomas-Fermi (TF) model to the outer reaches of the atom. With our derivation it can be seen that this is not necessarily correct, because the starting functional (1) is valid only when the gradient corrections are small compared with the main term ${ }^{10}$ and this is not true in the outer region of the atom. It is more adaquate to say that Eqs. (14) improve the TF model in the case that it is valid. This explains in part the poor results obtained by De Raad and Schwinger ${ }^{11}$ in the calculation of the magnetic susceptibility of diamagnetic atoms which, although improving the TF results, are far from experimental values. In a recent paper (published after the first submission of this Brief Report) Englert and Schwinger ${ }^{12}$ have shown that a modification of Eq. (14) greatly improves the results obtained for the magnetic susceptibility. We comment on the reason for that in the following.

According to our derivation, an improvement of Eqs. (14) should consist of calculating the corresponding equations to higher orders in $\epsilon$ and/or $\mu$. An equation correct to second order on $\epsilon$ should be rather involved and probably is not worthwhile due to the lack of convergence of the gradient expansion of the functional $E[\rho]$ in the outer regions of the atom. On the other hand, a consistent equation to second order in $\mu$ should contain, besides the exchange [last term of the first Eq. (13)], the correlation energy to first order. Nevertheless, at least for some properties, it may be more important to include exchange to second order than correlation to lowest order. An equation with these properties might be obtained by retaining only the desired terms of Eq. (5). However, a simpler procedure is to interpolate beween Eq. (14) and the Thomas-Fermi-Dirac (TFD) equation (which includes the exchange to an order higher than the first one). This means to search for an equation fulfilling two conditions: being equivalent to Eq. (14) to first order in both $\epsilon$ and $\mu$, and going to the TFD equation in the limit $\epsilon \rightarrow 0, \mu \rightarrow 1$. The solution is

$$
\begin{gathered}
\nabla^{2} \bar{V}=-(4 / 3 \pi)\left\{\left[-2 \bar{V}+(9 \mu+2 \epsilon)^{2} /(9 \pi)^{2}\right]^{1 / 2}\right. \\
+(9 \mu+2 \epsilon) / 9 \pi\}^{3} .
\end{gathered}
$$

This (with $\epsilon=\mu=1$ ) is just the equation successfully used by Englert and Schwinger ${ }^{12}$ for the calculation of the magnetic susceptibilities of atoms. A nontrivial problem is that of finding the appropriate boundary conditions, which we will not discuss here (see Ref. 12).

Summarizing, from any energy functional $E[\rho]$ it is possible in principle to derive an equation for the electric potential. In the regions with slowly varying and not too low density, the functional can be generated by means of an expansion in terms of two dimensionless parameters:

$$
\left(m e^{2} / \hbar^{2}\right) \rho^{-1 / 3}, \quad\left|\nabla\left(\rho^{-1 / 3}\right)\right|^{2} .
$$

These correspond to the formal parameters $\mu$ and $\epsilon$ introduced in this Brief Report. If the energy functional is known to a given order, $O\left(\mu^{n} \epsilon^{m}\right)$, the equation for the po- 
tential can be derived to the same order of accuracy. The lowest order-defined as zeroth, $O\left(\mu^{0} \epsilon^{0}\right)-$ is the early TF equation. The TFD equation is $O\left(\mu^{1} \epsilon^{0}\right)$, while Schwinger's equation ${ }^{9}$ is first order [i.e., it includes $O\left(\mu^{1} \epsilon^{0}\right)$ and $\left.O\left(\mu^{0} \epsilon^{1}\right)\right]$. The local density approximation is $O\left(\mu^{n} \epsilon^{0}\right)$ and Eq. (5) $O\left(\mu^{n} \epsilon^{1}\right)$, with $n$ limited by the knowledge of the density functional. Englert and Schwinger's equation (15) includes the exchange part of the terms $O\left(\mu^{2} \epsilon^{0}\right)$ besides the leading gradient correction $O\left(\mu^{0} \epsilon^{1}\right)$. To be consistent, it lacks the correlation energy term $O\left(\mu^{2} \epsilon^{0}\right)$, but this seems to be less important, as it is, probably, the gradient correc- tion to exchange, $O\left(\mu^{1} \epsilon^{1}\right)$. It must be pointed out again that no equation of the type discussed here is valid in the most external regions of the atom, due to the lack of convergence of the gradient density expansion. However, this difficulty may be mitigated with an adequate choice of boundary conditions.

We acknowledge useful comments of Dr. J. A. Alonso and Dr. L. C. Balbás. Partial financial support has been provided by the Comisión Asesora de Investigación Científica y Técnica, Madrid.
${ }^{1}$ P. Hohenberg and W. Kohn, Phys. Rev. 136, B864 (1964).

${ }^{2}$ See, e.g., A. L. Fetter and J. D. Walecka, Quantum Theory of Many-Particle Systems (McGraw-Hill, New York, 1971).

${ }^{3}$ See, e.g., H. Lewis, Phys. Rev. 111, 1554 (1958).

${ }^{4}$ A. S. Kompaneets and E. S. Pavlovskii, Zh. Eskp. Teor. Fiz. 31, 427 (1956) [Sov. Phys. JETP $\underline{4}, 28$ (1957)].

${ }^{5}$ D. A. Kirzhnits, Field Theoretical Methods in Many-Body Systems (Pergamon, Oxford, 1967).

${ }^{6}$ E. K. U. Gross and R. M. Dreizler, Z. Phys. A 302, 103 (1981)

${ }^{7}$ Yu. A. Borisov and V. V. Surikov, Zh. Strukt. Khim. 22, 164
(1981) [J. Struct. Chem. 22, 277. (1981)].

${ }^{8}$ E. H. Lieb, Rev. Mod. Phys. 53, 603 (1981).

${ }^{9}$ J. Schwinger, Phys. Rev. A 24, 2353 (1981).

${ }^{10}$ See, e.g., D. C. Langreth and J. P. Perdew, Solid State Commun. 31, 567 (1979); J. A. Alonso and L. A. Girifalco, Phys. Rev. D

$\underline{17}, 3735$ (1978); O. Gunnarsson, M. Jonson, and B. I. Lundqvist, Phys. Rev. B 20, 3136 (1979).

${ }^{11}$ L. L. De Raad and J. Schwinger, Phys. Rev. A 25, 2399 (1982).

${ }^{12}$ G. B. Englert and J. Schwinger, Phys. Rev. A 26 , 2322 (1982). 